\title{
Adaptive Seeking Control for Magnetic Disk Drives*
}

\author{
Masahito KOBAYASHI**, Takashi YAMAGUCHI** \\ and Hiromu HIRAI**
}

\begin{abstract}
The digital servo system of a magnetic disk drive has to overcome poor seek control performance caused by the mechanical gain variations and force disturbance variations in each head. This paper presents an adaptive seek control strategy which adjusts the acceleration feedforward gain to minimize the square of the velocity error. The adaptive seek control is implemented using a digital signal processor and its control performance is good.
\end{abstract}

Key Words: Adaptive Control, Positioning, Digital Control, Magnetic Disk Drive, Seeking Control

\section{Introduction}

Magnetic disk drives are used for storing relatively large amounts of information that can be accessed in milliseconds. It is necessary to improve the performance of the head-positioning mechanism in order to increase track density for achieving more storage capacity and reduce access time for enhancing data throughput. The head-positioning servo has two main modes : track following and track seeking. The track following mode accurately maintains the selected head position along the center of the track. The track seeking mode provides rapid movement of the head from one track to another selected track.

Generally, a velocity feedback controller is used in the track seeking mode to follow a predetermined reference velocity profile. The reference velocity profile, stored as a table in the microprocessor, is a function of the remaining distance between the current and target tracks. When the head nears its target track, control is switched from the track seeking controller to the track following controller. This

* Received 4th October, 1999

** Mechanical Engineering Research Laboratory, Hitachi, Ltd., 502 Kandatsu, Tsuchiura, Ibaraki 300-0013, Japan. E-mail : masahito@ merl.hitachi.co.jp transition, called the settling period, has a big impact on the servo performance in terms of both access time and positioning accuracy. Using a simple proportional gain feedback, a constant velocity error will occur when the reference profile is a ramp during the deceleration period of the seeking mode. A twodegree-of-freedom controller which has an acceleration feedforward profile, can be used to eliminate the velocity error. The acceleration feedforward signal is determined by differentiating the velocity profile and then adding it to the velocity error signal after gain feedback compensation. As the feedforward controller compensates the delay of the feedback controller, the seek controller with the acceleration feedforward makes the head accurately follow the reference velocity profile without increasing the servo bandwidth or using a slow reference velocity profile. Thus, the velocity error is small when the controller switches to the following mode and a good response for the target track is obtained during the settling period.

The servo performance, however, changes due to manufacturing tolerances, time, and environmental conditions. Under recent narrow track pitches, the problems of gain variation, as well as bias force, have become impossible to neglect. The actuator and heads are electro-mechanical components and their parameters are subject to variation. Depending on the 
actuator position, the actuator torque constant varies by about $10 \%$ because of the non-uniform flux density. The torque constant is small when the head is located at the inner and outer sides of the disk. The position detector constant of each head varies due to the manufacturing tolerance of the core width. In addition, other system parameters, such as inertia, A/D converter gain, $\mathrm{D} / \mathrm{A}$ converter gain, and servo power amplifier gain can vary. Also, typical bias forces, which vary with the radial actuator position, are caused by the flexible printed cable to the actuator, friction of the pivot ball bearing of the rotary actuator and electrical offsets in the circuit. The servo performance shifts away from optimal performance due to these plant uncertainties. The feedforward controller based on the nominal plant model is particularly sensitive to the model uncertainties described above. Thus, the deceleration velocity profile is generally designed to cope with worst case variations. At the end of the deceleration phase, the head moves exponentially with a slow-slope so that each disk drive has a smooth settling response. This is one reason that the access time increases under nominal conditions.

The servo controller has to overcome poor servo performance caused by the gain variations and bias force of the plant in each head and each track. The main feature of the adaptive control schemes is to maintain a specified nominal performance in the presence of gain variations ${ }^{(1)}$. Recently, several papers have described control designs with adaptation for magnetic disk drives or optical disk drives ${ }^{(2)-(6)}$. Most of the papers focus on track following mode adaptation.

This paper presents a new, on-line adaptive seeking controller which estimates plant parameters during the seeking mode so as to eliminate the initial state variation at the start of the settling period. The adaptive seeking controller sequentially adjusts the controllable variable gain of the reference acceleration signal, so that the velocity error caused by parameter gain variation and bias force is minimized.

\section{Adaptive Seeking Control Design}

\section{1 Basic structure}

Figure 1 shows the structure of the adaptive seeking control with an adaptive adjustment mechanism that uses the gradient method. The adaptive adjustment mechanism sequentially adjusts the variable gain $\theta(k)$ of the acceleration feedforward signal in order to minimize the square of the velocity error, $e_{v}^{2}(k)$, during the deceleration seeking period at each sampling instant $k$. When $e_{v}^{2}(k) \rightarrow 0$ is achieved, the loop gain $\theta / k_{m 0}$ of the acceleration feedforward path is

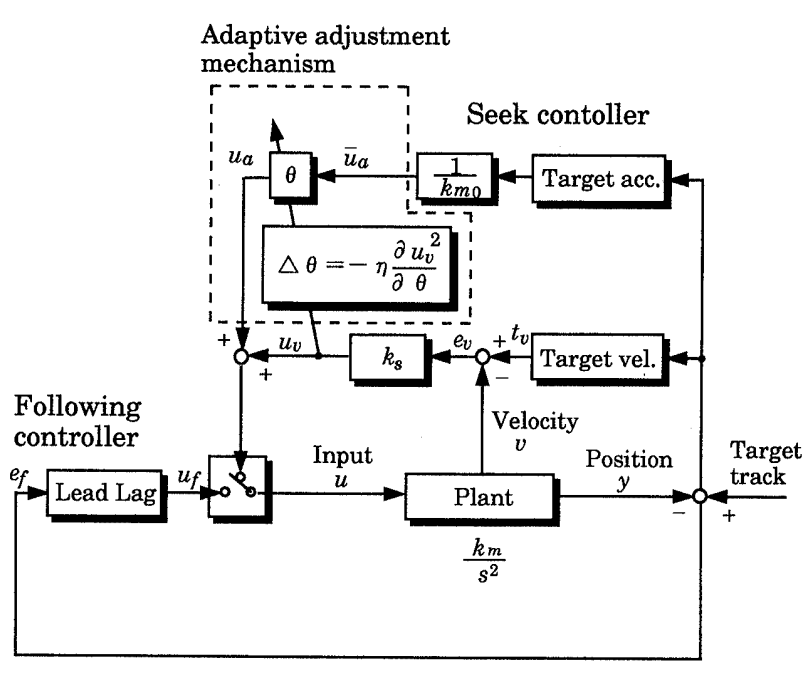

Fig. 1 Structure of the adaptive seeking control

equal to the inverse loop gain $1 / k_{m}$ of the plant. Here, $k_{m}$, the loop gain of the plant from control input, $u$, to velocity output signal, $v$, consists of the $\mathrm{D} / \mathrm{A}$ converter gain, power amplifier gain, force constant of the voice coil motor, and inertia. $k_{m 0}$ represents the nominal value for $k_{m}$. Note that if $k_{m}$ is known, then $k_{m 0}$ would logically be chosen to be $k_{m}$.

The performance index to be minimized is

$$
\begin{aligned}
& E(k)=\frac{1}{2} u_{v}^{2}(k) \\
& \quad=\frac{1}{2}\left(u(k)-u_{a}(k)\right)^{2}
\end{aligned}
$$

where $u(k)$ is control input, $u_{a}(k)$ is acceleration feedforward signal, and $u_{v}(k)$ is the feedback control input, defined as

$$
u_{v}(k)=k_{s} \cdot e_{v}(k)
$$

with velocity feedback gain $k_{s}$.

The variable estimation gain $\theta(k)$ of the acceleration feedforward is related as

$$
u_{a}(k)=\theta(k) \bar{u}_{a}(k)
$$

where $\bar{u}_{a}$ denotes the input signal to the variable gain of the acceleration feedforward signal. The estimation gain $\theta(k)$ is updated in order to minimize the performance index using the adaptive gradient law, that is,

$$
\begin{aligned}
& \theta(k+1)=\theta(k)-\eta \frac{\delta E(k)}{\delta \theta(k)} \\
& \quad=\theta(k)-\eta \frac{\delta E(k)}{\delta u_{a}(k)} \cdot \frac{\delta u_{a}(k)}{\delta \theta(k)} \\
& =\theta(k)+\eta \cdot\left(u(k)-u_{a}(k)\right) \cdot \bar{u}_{a}(k) \\
& =\theta(k)+\eta \cdot u_{v}(k) \cdot \bar{u}_{a}(k) .
\end{aligned}
$$

where $\eta$ denotes the adaptation gain. The initial estimate for $\theta(0)$ should be set 1.0. The adjustment does not update during the acceleration period because the actuator moves due to the maximum saturated voltage. 


\section{2 Properties of the adaptive algorithm}

This section discusses the convergence properties of the velocity error using the gradient algorithm. At first, the estimation equation is introduced. In Fig. 1, the plant transfer function from control input $u$ to head velocity $v$ is given as,

$$
v(k)=k_{m} Z\left[\frac{1}{s}\right] u(k)
$$

where $k_{m}$ is the plant loop gain, $s$ denotes a Laplace operator, and $Z[\cdot]$ indicates a $z$ transform. The acceleration feedforward signal $\bar{u}_{a}$ is designed by differentiating the reference velocity profile, such that,

$$
\bar{u}_{a}(k)=\frac{1}{k_{m 0}} Z[s] t_{v}(k)
$$

where $t_{v}$ is the reference velocity profile. As the aim of the velocity control is $v=t_{v}$, substitute equation ( 5 ) into Eq. (6),

$$
\begin{gathered}
\bar{u}_{a}(k)=\frac{Z[s]}{k_{m 0}} \cdot \frac{k_{m}}{Z[s]} u(k) \\
\triangleq \frac{1}{\theta_{0}} u(k)
\end{gathered}
$$

where $\theta_{0}$ is an unknown parameter which is defined by

$$
\theta_{0} \triangleq \frac{k_{m 0}}{k_{m}} \text {. }
$$

In this case, $k_{m 0}$ should be designed as $k_{m 0}=k_{m}$.

Hence, the ideal estimation equation of the plant without perturbation is

$$
u(k)=\theta_{0} \bar{u}_{a}(k) \text {. }
$$

The estimation equation used during estimation is given by

$$
u_{a}(k)=\theta(k) \bar{u}_{a}(k) .
$$

The input signal $u$ is

$$
u(k)=u_{a}(k)+u_{v}(k)
$$

The estimation error between Eqs. ( 8$)$ and (9) is

$$
\begin{aligned}
& u_{v}(k)=u(k)-u_{a}(k) \\
& \quad=\left(\theta_{0}-\theta(k)\right) \bar{u}_{a}(k) \\
& \quad \triangleq \phi(k) \bar{u}_{a}(k)
\end{aligned}
$$

where

$\phi(k) \triangleq \theta_{0}-\theta(k)$.

Next, the principle of the adaptive law is discussed. The resulting update law in Eq. (4) is rewritten using Eq. (11) as

$$
\begin{aligned}
& \theta(k+1)=\theta(k)+\eta \cdot\left(\theta_{0}-\theta(k)\right) \bar{u}_{a}^{2}(k) \\
& \quad \triangleq \theta(k)+m .
\end{aligned}
$$

The meaning of the above equation can be described below.

- If $\theta_{0}=\theta(k)$, then $\theta(k+1)=\theta(k)$.

- If $\theta_{0}>\theta(k)$, then $m>0$. Which means the law updates $\theta(k+1)$ to the correct value.

- If $\theta_{0}<\theta(k), m<0$. Which means the law updates $\theta(k+1)$ to the correct value.

It is easy to see that Eq. (11), based on the gradient law, can decrease the performance index.

Subtracting the unknown parameter $\theta_{0}$ from both sides of Eq. (4) results in

$$
\phi(k+1)=\phi(k)-\eta \cdot u_{v}(k) \cdot \bar{u}_{a}(k)
$$

Let the Lyapunov candidate function be

$$
V(k)=\phi(k)^{2} \text {. }
$$

This Lyapunov candidate function has the special form of the norm square of the identifier parameter error. Its increments are calculated as follows,

$$
\begin{aligned}
& \Delta V(k)=V(k+1)-V(k) \\
& \quad=-\eta \cdot u_{v}^{2}(k)\left(2-\eta \cdot \bar{u}_{a}^{2}(k)\right) .
\end{aligned}
$$

That is, $\Delta V(k) \leq 0$ is achieved as long as

$$
0<\eta<\frac{2}{\bar{u}_{a}^{2}(k)} \text {. }
$$

In the case of the seeking control system of a magnetic disk drive, the acceleration feedforward is used to guide the head along the reference velocity profile during the deceleration period so the acceleration feedforward signal is always less than zero, that is $\bar{u}_{a}(k)<0$. It is possible to find a value for $\eta$ in order to satisfy Eq. (16). $V(k)$ decreases with respect to $k$. So, $\Delta V(k)$ is a Lyapunov function for the overall system. Hence, the velocity error $u_{v}(k)$ converges to zero,

$$
\lim _{k \rightarrow \infty} u_{v}(k)=0 \text {. }
$$

Moreover if $\bar{u}_{a}(k)$ satisfies the P.E. condition, then

$$
\lim _{k \rightarrow \infty} \theta(k)=\theta_{0}
$$

\section{3 Adaptive bias force estimation mechanism}

Adaptive bias force estimation is introduced by adding another variable estimation gain $\theta_{b}(k)$ for the bias force. In this case, the control input is

$$
u(k)=u_{v}(k)+u_{a}(k)+u_{b}(k)
$$

and the estimation gain $\theta_{b}(k)$ is related as

$$
u_{b}(k)=\theta_{b}(k) \cdot \bar{u}_{b}(k)
$$

where $\bar{u}_{b}(k)=1.0$. The adaptive estimation law to minimize Eq. (1) is established by both Eq. (4) and

$$
\begin{array}{r}
\theta_{b}(k+1)=\theta_{b}(k)-\eta_{b} \frac{\partial E(k)}{\partial \theta_{b}(k)} \\
=\theta_{b}(k)+\eta_{b} \cdot u_{v}(k) \cdot \bar{u}_{b}(k)
\end{array}
$$

where $\eta_{b}$ denotes the adaptation gain and the initial estimate for $\theta_{b}(0)=0.0$. During the deceleration period of the seeking mode, both Eqs. (4) and (21) are calculated. If an accelerometer device can be attached at the base plate of the magnetic disk drive, the acceleration signal from the accelerometer can be used instead of $\bar{u}_{b}(=1.0)$ to reject the external disturbances.

\section{Experimental Results}

The adaptive controller described in the previous chapter was implemented in a magnetic disk drive with a digital control set-up shown in Fig. 2. The digital controller was based on software routines implemented in a 32-bit TMS320C30 (TI) Digital Signal Processor (DSP). The sampling period is $50 \mu \mathrm{s}$. 


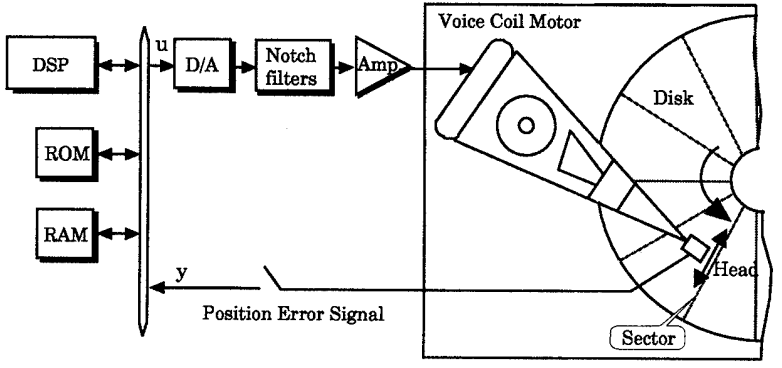

Fig. 2 Experimental equipment

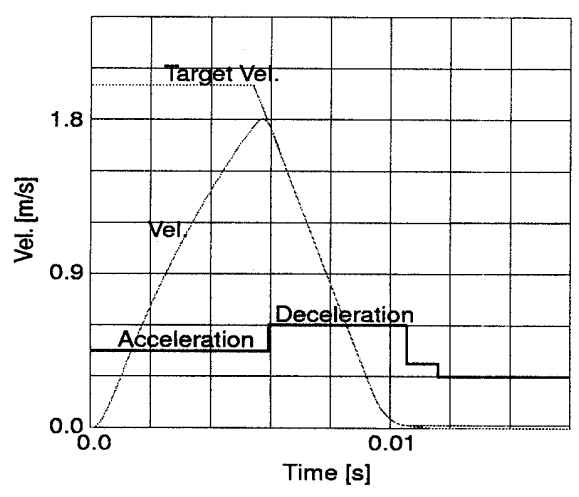

(a) Velocity and target velocity

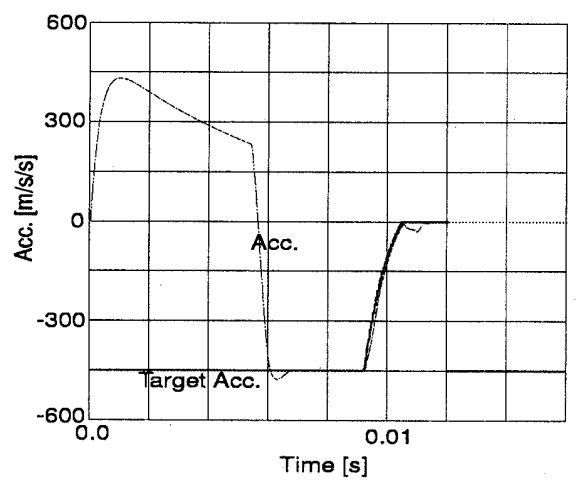

(b) Acceleration and target acceleration

Fig. 3 Response waveforms of the conventional seek control (Simulation results)

The output signal of the $\mathrm{D} / \mathrm{A}$ converter is passed through notch filters $(2 \mathrm{kHz}, 4 \mathrm{kHz})$ to eliminate mechanical resonances and fed into the amplifier which drives the voice coil motor. The head moves to the target track in response to the voice coil motor. The magnetic head reads the pre-recorded servo information stored on the disk at each sampling period. The position error signal (PES) detected by the position detector indicates the position error of the head away from the nearest track centerline.

The cross-over frequency of the seek controller is $\omega_{x}=350 \mathrm{~Hz}$ and the velocity signal $v$ is calculated by the backward difference method. Figure 3 shows the simulation results of the conventional $1 / 3$ stroke seek response. In the figure, both the target velocity profile and the target acceleration profile are shown. The maximum target acceleration is $450 \mathrm{~m} / \mathrm{s}^{2}$ and the maximum target velocity is $2.0 \mathrm{~m} / \mathrm{s}$. The mode is switched from the seek controller to the following controller when the head is within 1 track of the target track. The cross-over frequency of the following controller is $\omega_{x}=500 \mathrm{~Hz}$.

The responses of the $1 / 3$ stroke seek with $\pm 15 \%$ gain variation are compared. The gain variation is changed during the deceleration period using software. Figure 4 shows the conventional seek control response. In the figure, Cur indicates the current signal of the voice coil motor, Vel denotes the velocity signal and Pos indicates the head position signal. If the gain decrease is $-15 \%$, as in Fig. 4 ( a ), the head overshoots during the settling period because of less stopping ability. If the gain increase is $+15 \%$, as in Fig. 4(c), the head has a good response but access time is much longer than the nominal condition shown in Fig. 4 ( b ).

Figure 5 shows the adaptive seeking control response with $\pm 15 \%$ gain variation. The adaptation gain of the adaptive seek system is $\eta=0.0000006$. The initial estimation value is $\theta(0)=1.0$. In Fig. $5(\mathrm{a})$, the real value of $\theta_{0}$ is 1.18 (i.e., $1 / 0.85$ ). In Fig. 5 ( c ), the real value of $\theta_{0}$ is 0.87 (i.e., $1 / 1.15$ ). The estimated value of $\theta$ in both figures converges accurately to the real value within $4 \mathrm{~ms}$ and the acceleration feedforward with adaptation gain can compensate for the gain variation of the plant. The position responses shown in both Figs. 5(a) and (c) are almost the same as the nominal response in Fig. $5(\mathrm{~b})$. When $\pm 20 \%$ gain variations are applied to the plant, good performance could still be obtained by using the adaptive seek controller. Without adaptation, however, seeking control error occurs by using the conventional seek controller.

At the beginning of the settling period, the initial state distributions of both head velocity and position are measured under the random seek operation from 1 track seek to $1 / 3$ track seek. Comparisons of the initial state distributions under gain variation of $\pm 10 \%$ without adaptation are shown in Fig. 6 and with adaptation are shown in Fig. 7. These figures show that the adaptive control method improves the distribution to become nominal one.

\section{Conclusion}

The servo controller should maintain access reliability at a high level. The adaptive controller based on the gradient law adjusted the acceleration feedforward gain to minimize the square of velocity error. This was successfully implemented on a digitally 


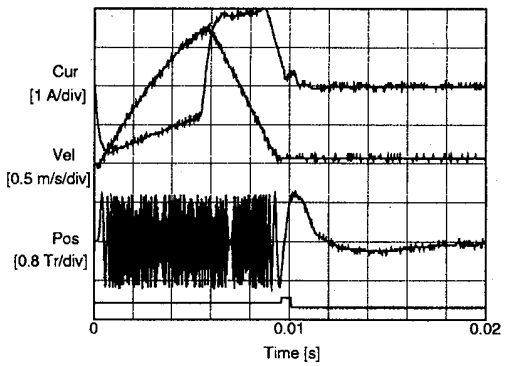

(a) Gain variation $-15 \%$

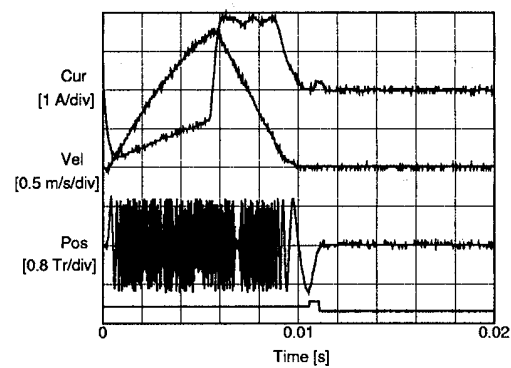

(b) Gain variation $0 \%$

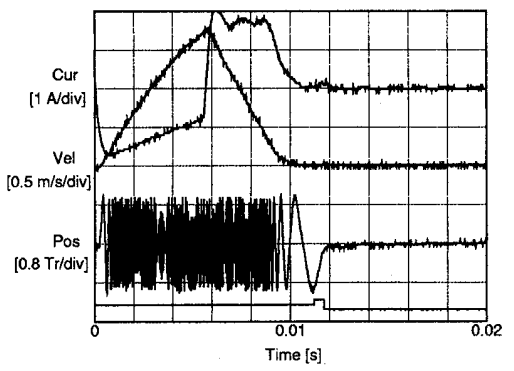

(c) Gain variation $+15 \%$

Fig. 4 Response waveforms of the conventional seek control (Experimental results)

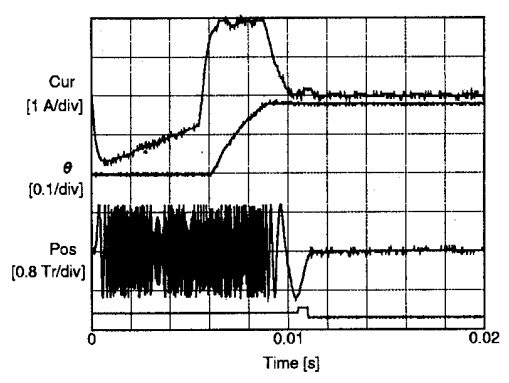

(a) Gain variation $-15 \%$

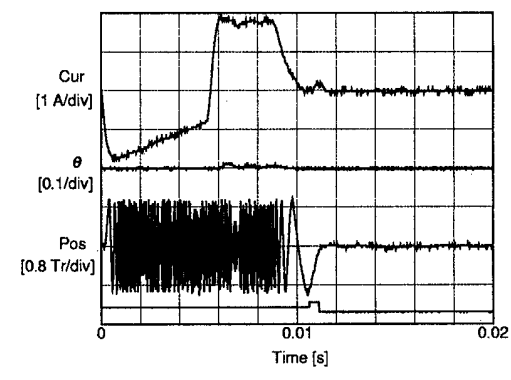

(b) Gain variation $0 \%$

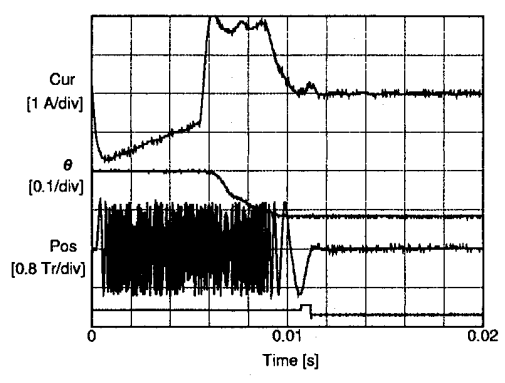

(c) Gain variation $+15 \%$

Fig. 5 Response waveforms of the adaptive seeking control (Experimental results)

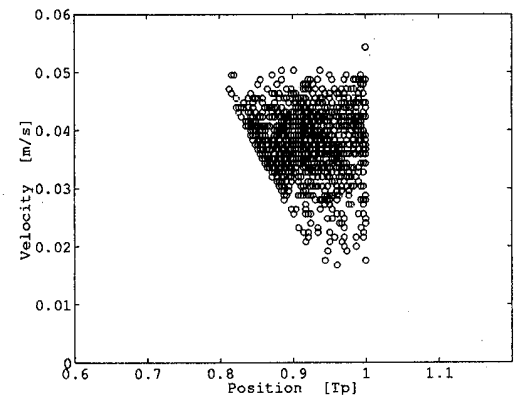

(a) Gain variation $-10 \%$

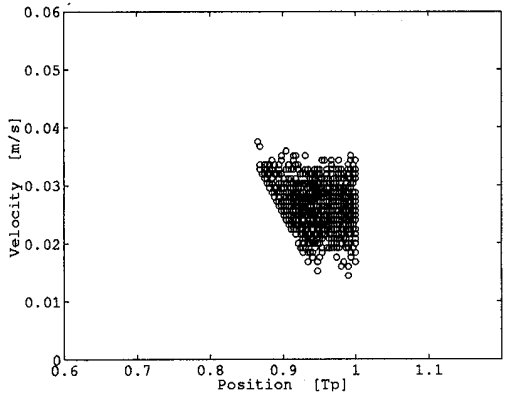

(b) Gain variation $0 \%$

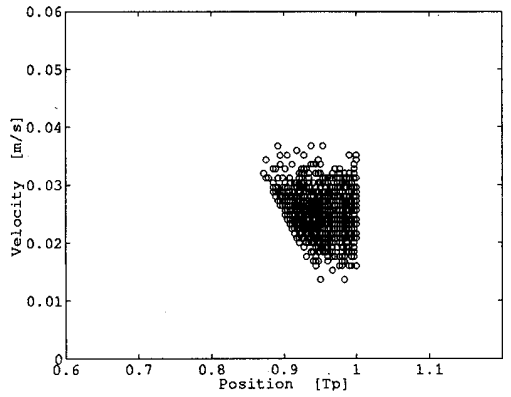

(c) Gain variation $+10 \%$

Fig. 6 Initial states at the beginning of the settle period of conventional seek control

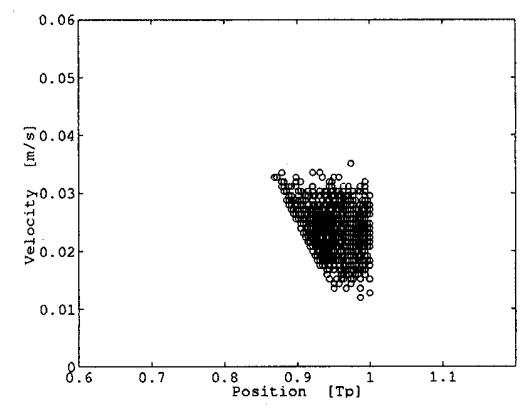

(a) Gain variation $-10 \%$

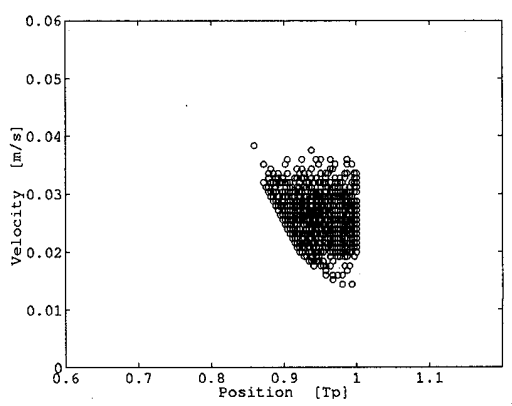

(b) Gain variation $0 \%$

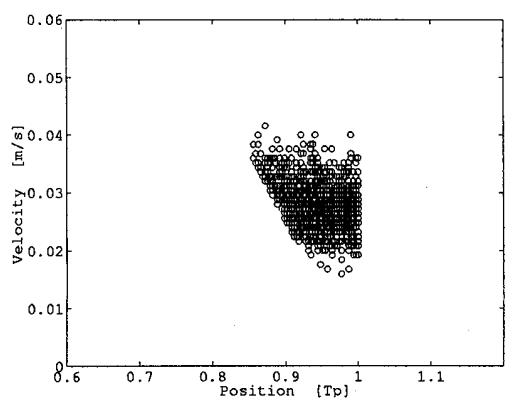

(c) Gain variation $+10 \%$

Fig. 7 Initial states at the beginning of the settle period of adaptive seeking control 
controlled magnetic disk drive. The implemented real -time adaptive method gave a high-speed adaptation and an accurate estimation during the deceleration seek period. This was achieved without any additional external excitation or specific calibration routine. The experimental results showed that the performance with adaptation for the track seeking response could be uniform under plant gain variations.

\section{References}

(1) Åström, K.J. and Wittenmark, B., Adaptive Control, (1995), Addison-Wesley.

( 2 ) Franklin, G.F., Powell, J.D. and Workman, M.L., Digital Control of Dynamic Systems, Chapter 11, (1990), Addison-Wesley.
( 3 ) Messner, W. and Horowitz, R., Identification of a Nonlinear Function in a Dynamical System, Trans. ASME Journal of Dynamic Systems, Measurement, and Control, Vol. 115 (1993), p. 587-591.

(4) Draijer, W., Steinbuch, M. and Bosgra, O.H., Adaptive Control of the Radial Servo System of a Compact Disc Player, Automatica, Vol. 28, No. 3 (1992), p. 455-462.

(5) Horowitz, R. and McCormick, J., A Self-Tuning Control Scheme for Disk File Servos, IEEE Trans. Magnetics, Vol. 27, No. 6 (1991), p. 4490-4495.

(6) Kobayashi, M., Yamaguchi, T. and Hirai, H., Adaptive Control of a Hybrid Servo System for Magnetic Disk Drives, Proceedings of the 1993 JSME International Conference on Advanced Mechatronics, (1993), p. 725-730. 